\title{
Trial designs for vaccines adapt to the times
}

The trial protocols for diseases common to the developed world now routinely change in midstream in response to real-time analysis of subject data. Yet such 'adaptive' designs have been slower to catch on for infectious disease trials. "It's great that oncologists are using it to develop new treatments," says Trudie Lang, head of the Global Health Trials program at the University of Oxford's Centre for Tropical Medicine in the UK. "But where we could really make a difference and speed things up is to use it in the global health arena, and we're not."

In December, Lang put out a call for more researchers studying neglected diseases to implement adaptive trial designs. Her reasoning, put forth in a commentary, is that resource-limited settings have the most to gain from flexible protocols, which can make trials more cost effective (Am. J. Trop. Med. Hyg. 85, 967-970, 2011). "This is not about cutting corners," Lang says. "It's about getting the right answer in the shortest period of time and with the least money."

Her plea has not fallen on deaf ears. Late last year, Aeras, a nonprofit based in Rockville, Maryland that develops products against tuberculosis, made the largest preapproved, real-time protocol change to date for any infectious disease vaccine trial. Together with Crucell, a Dutch biopharmaceutical company, the organization is testing out Aeras-402, an experimental tuberculosis vaccine that it designed to boost immunity in people who have already received bacillus CalmetteGuérin (BCG), the only vaccine currently available for protection against the lung disease.

After examining three different dosing amounts of Aeras-402 as well as of placebo in around 200 children at three sites in Africa, the organizers took a planned look at the immune response and safety profiles in the infants and decided to move forward with only the highest dose originally given.

"This is a very exciting trial," says Sharon Nachman of the Stony Brook University Medical Center in New York State, who vicechairs the International Maternal Pediatric Adolescent AIDS Clinical Trials group's tuberculosis scientific committee, which is cosponsoring the trial. "The fact that we've got this flexibility to say 'let's look at it, let's think about it and let's fix it' is really huge."

Aeras and Crucell now plan to affirm the safety of the chosen dose in a larger cohort of 250 infants and then move into the main phase of the innovative study: enrolling anywhere from 1,950 to 3,750 more trial participants, depending on the site-specific infection rates that the investigators observe on the ground. "In the past when we had these very inflexible study designs imposed by the statisticians and the regulatory authorities, we often enrolled a lot of people, put in a lot of work and didn't get anything out of it-and that didn't help anybody," says Aeras' chief scientific officer Tom Evans. "What we're starting to do now is assure the community that the trials we're conducting will be sufficient to get the answers to the questions we're asking."

The global health community seems to be taking notice. Adaptive trials are "really the way the forward in this area," says Gerry Davies, a tuberculosis researcher at the University of Liverpool, UK. “There's evidence that people are starting to take it up, there's enthusiasm among people who are powerful in the field and there's engagement with regulators that could make it happen. So, it's coming."

\section{In the interim}

HIV vaccine trials could be the next to adopt such innovative trial designs. For instance, the HIV Vaccine Trials Network, an international group sponsored by the US National Institute of Allergy and Infectious Diseases, is gearing up to test multiple candidate vaccines in parallel against a common placebo with sequential monitoring. With each regimen, a first stage will evaluate the vaccine's preliminary efficacy over 18 months, and only those products that show promise in protecting against infection will stay in the trial for the extra three years needed to demonstrate lasting immune responses.

Although a modified version of the vaccine that showed around $30 \%$ protection in the so-called 'Thai trial' will probably reenter phase 3 testing, no obvious backup product has yet emerged as the next best candidate. Organizers of the adaptive phase $2 \mathrm{~b}$ approach say the trial design should help the field focus its resources on only the most promising vaccines-and yield important lessons along the way. "The point is to get results in the field faster, to keep the progress moving and hopefully get to an approved vaccine faster," says Peter Gilbert, a biostatistician at the Fred Hutchinson Cancer Research Center in Seattle who is spearheading the trial design. "We're learning fundamental science about how one or more of the vaccines seem to work and then synthesizing all of that to come up with a plan to develop a new vaccine regimen."

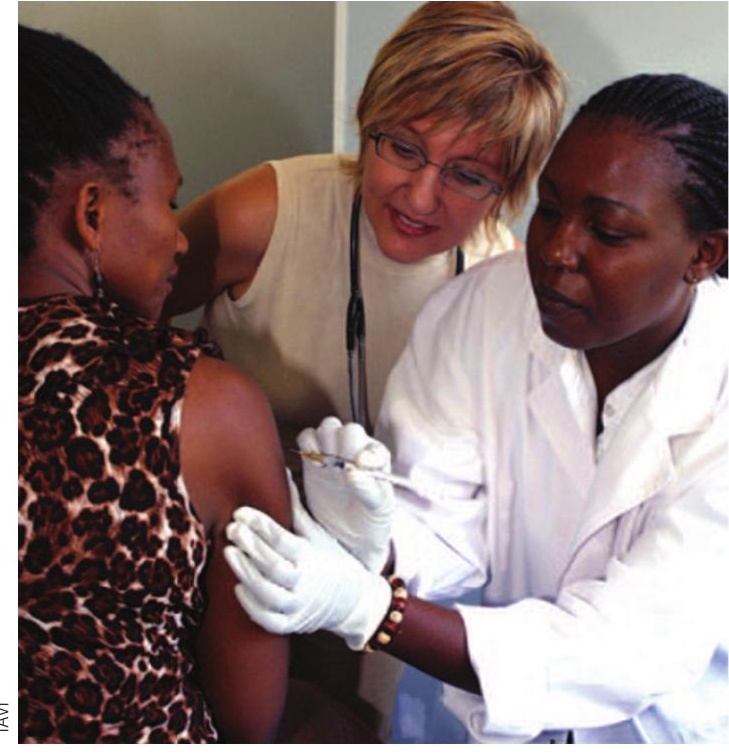

Adapting to change: Vaccine trials come around.

"It's surprising to me that it's never been done before," Gilbert adds.

Despite all the planning going into adaptive vaccine designs, however, they still don't always work out as intended. The Aeras-402 study, for example, included prospectively planned opportunities to modify the vaccine dosing, the enrollment numbers and the duration of follow-up - foresight that is required by ethics boards and regulatory bodies for trial sponsors to seamlessly change protocol in midstride. Nonetheless, earlier this year the organizers sought to make an unscheduled change to the protocol. "It's one of these trials where we're learning as we go," says Aeras' senior medical advisor Lewellys Barker.

Off the back of a small phase 1 trial in infants completed two years ago, the AerasCrucell team had designed the two-shot approach for their large adaptive study. But the trial investigators decided in January to consider adding a third booster shot going forward to improve the immune response. This additional step was not written into the approved protocol, and so the amendment, which was still being finalized as Nature Medicine went to press, will need to be cleared by all the ethics boards and regulatory agencies involved in the study.

Even so, Evans remains confident that the protocol change will be approved before any child in the study is ready to receive his or her booster shot. "The trial is rolling along as originally planned," he says.

Elie Dolgin 